\title{
Prospects for Computer-Aided Design of Castings
}

\author{
Volodymyr Doroshenko ${ }^{1}$, Olena $\mathrm{Mul}^{2}$, Olena Kravchenko ${ }^{3}$, \\ ${ }^{1}$ Physico-Technological Institute of Metals and Alloys, \\ ${ }^{2}$ Ternopil National Ivan Puluj Technical University, \\ ${ }^{3}$ International Research and Training Center for Information Technologies and Systems
}

\begin{abstract}
In the study on limiting possibilities of a casting process in terms of resource saving it is considered which solidstate constructions can optimally fill the space of the mold. The possibility of designing patterns for the Lost Foam Casting process in accordance with mathematical concepts is shown. The examples of patterns of frame cellular castings are described. The examples of cellular castings, designed with the help of computer technologies, are shown that often inherit structures of nature. Such castings allow expanding of the existing range of properties of metal products.
\end{abstract}

Keywords - Cellular castings, computer simulation, engineering castings, foam patterns, gyroid, lost foam casting, mathematical concepts, minimal surfaces, structures of nature, quasicrystals.

\section{INTRODUCTION}

Lost Foam Casting (LFC) into a vacuumized sand mold provided a new look at the metal casting design.

On the one hand, the material of the used foam pattern on a thin section looks under a microscope like a small-cell foam, wherein air is "packed" into thin polystyrene shells and the metal, poured onto the pattern, gasifies these shells and replaces the pattern.

On the other hand, dry sand under vibro- and/or pneumoimpact during the molding process is similar in properties to the "pseudo-liquid", which flows around the pattern. Then the casting pattern can be considered as a construction for the impact of two fluids: the first is the metal, which replaces the pattern from the inside while pouring; the second is the "pseudo-liquid" of sand, which flows around the pattern surface outside during molding.

During research of limiting possibilities of the casting process and optimization of casting constructions in terms of resource saving the following question arose: which solid-state constructions could optimally fill the space, occupy the volume of the mold sand and "spill" in it?

The first clues for the answer are the constructions observed in animate and inanimate nature, as well as models of microstructures of organic and inorganic substances in the form as they are represented in the modern natural sciences.

The main difference between the engineering constructions, proposed by human, and those created by nature is the high energy efficiency of the last ones. Also, in many cases, the difference between them is in the presence of such characteristic properties as repeatability of identical elements in different directions, combinatority (fractality) and cellularity.

In particular, bionics studies the use of organization principles, properties, functions and structures of animate nature in technical devices. However, in engineering, i.e. creating a prototype of some supposed or possible object, there can be used not only images of animate and inanimate nature but also imaginary, ideal, for example, mathematical models, which in general case do not necessarily have accordance with something in the physical world.

\section{ANALYSIS OF THE LATEST INVESTIGATIONS}

This article continues the coverage of examples of both engineering cast frame cellular products and the search for ways to optimize their structure. That includes borrowing "technical solutions" from nature, which solved problems of the space "conquest" by constructions with high efficiency and resource saving.

Earlier the possibility of copying the structures of the simplest crystal lattices in cast products using volumetric prefabricated structures with repetitive unified elements has been described in [1]. The foam patterns of such elements can be produced at automatic formation machines or 3D-mills with the help of computer programs. The simplest and most noticeable in their properties structures were taken as the analogs for engineering the above cast constructions.

At present, computer simulation helps to reflect our conceptions about the world structure and borrow from it some of the details for our own man-made constructions. The basis of our knowledge of chemistry, physics, materials science, earth sciences and others is mainly the knowledge about the substance structure, which largely determines the substance properties. Thus, there arose the problem of creating new materials by calculating their structures on the computer.

Until recently, the problem of predicting the crystal structures on the basis of the chemical composition was considered unsolvable. Traditionally, inventions of new materials for the needs of engineering occurred either by the trial and error method, or just by chance. At present, thanks to the sufficiently high level of development of the quantum theory of materials, creation of new materials with the help of computers has become possible [2].

The stable crystal structure is characterized by the lowest energy. This problem can be solved by investigating all possible relative positions of atoms, calculating the energy for each of them and in this way determining the lowest energy and the optimal structure.

Complexity of the problem lies in the fact that the number of variants in the structure is astronomically large. Directly, this problem is unsolvable. However, it can be solved without exhaustive search but by directing calculations, with the help of self-training, to the global minimum of energy. In this vein, 
the approach is developed, based on the ideas of evolution, which is a multidimensional minimization for the search of any thermodynamically stable states [2].

Creation of data analysis methods led crystallographers to the field of multidimensional geometry. Also, similar data analysis, in fact by means of multi-dimensional geometry, is carried out by anthropologists and literary critics. Thus, similar methods are long used by literary critics to determine the authorship of texts as well as by geneticists and anthropologists to determine the kinship among nations based on DNA.

\section{DISCUSSION AND RESULTS}

At a junction of different sciences, similar ideas can be applied to a variety of scientific fields. If programs of designing structures of new materials from atoms are created, then by copying the atomic lattices analogically to the examples in [1] one can get their macro-sized foam patterns for spatial lattice castings and develop methods of casting such cellular metal products.

Expanding the range of castings designs and LFC-process allows to open a new direction of casting cellular, volumecellular and skeletal lattice metal products. Such products have the potential for use as lightweight carriers, reinforcing, insulating, protecting and damping loads spatial constructions, which can absorb or pass through their cells some flow of matter or energy.

The concept of simplification of assembly of cellular casting foam patterns into volumetric structures by using repetitive unified elements of serial production has analogues in various mathematical models.

The whole class of periodical minimal surfaces (MS) can be obtained by repetition of some unit cell. The unit cells of many of such minimal surfaces, in turn, can be "assembled" from the copies of some fundamental element and its specular reflection.

The theory of MS is one of the classical and at the same time developing areas of mathematics at the junction of geometry, topology and calculus of variations. A soap film is the obvious realization of MS: it tightens the contours of different configuration and takes the shape, corresponding to the minimum of potential energy (surface tension energy), which is directly proportional to its area.

One of the remarkable examples of infinitely connecting and repetitive in three dimensions structure with MS is gyroid. It was first described in 1970 by the American physicist Alan Schoen from NASA, who was looking for the ultra-light and durable material for spacecrafts [3].

The surface of the gyroid is described by the following equation:

$$
\cos (x) \cdot \sin (y)+\cos (y) \cdot \sin (z)+\cos (z) \cdot \sin (x)=0 .
$$

Its sample, obtained in bronze by the investment casting method by sculptor and mathematician Bathsheba Grossman [4], is shown in Fig. 1a).

The reduced jewelry version of the same authorship is produced using a 3D-printer.

Bodies with the gyroid structure are present in the cells of plants and animals in separate block copolymers at condensation of oligomers.

Scientists explain patterns on the palms, swollen of water, by the gyroid organization of the "skeleton" of the folds from the keratin fibers, which are laid in the skin as if they were in the gyroid cavities.

The gyroid structure (Fig. 1b)) is shown on the website of the Institute of Polymer Science of the University of Ulm [5].

The pattern for the casting in Fig. 1a) is obtained by the assembly from repetitive plates in the form of regular hexagons with a contoured surface, having convex and concave parts. Such plates can be called the fundamental elements. Their shape of a regular hexagon allows it to make them of two, three or six pieces as of repetitive elements in combination with copies of their "specular reflections".

The pattern with the gyroid surface can be assembled without size limitations, using such fundamental elements of only one type.

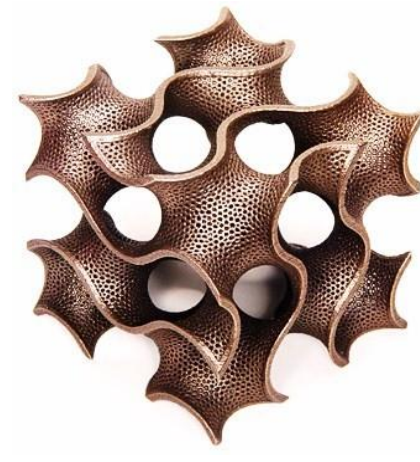

a)

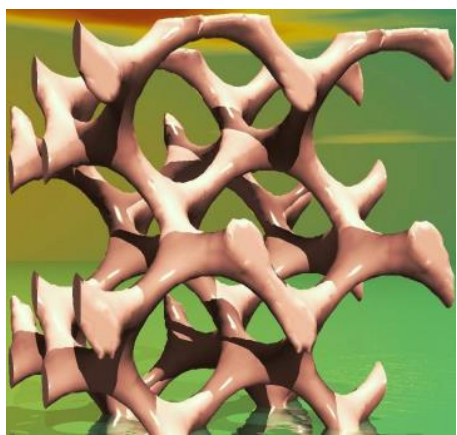

b)
Fig. 1. Samples of constructions with the gyroid structure: a) metal casting; b) polymer structure.

For the small-scale production of foam patterns such plates or their above mentioned components can be performed flat and only after being bent by the method [6].

For the serial production of the described patterns it is more effective to produce their repetitive elements at automatic formation machines or in press molds by the autoclave method and then assemble by the known methods: by gluing, by the tongue and groove system and by others.

The casting pattern with MS during molding interacts with sand on the minimum area of its surface, rubbing against the sand, which contributes to the uniform sand compaction.

The MS presence for single patterns increases the number of options for their technological arrangement in the sand of the container mold without the risk of getting undercuts and channels, unfilled by sand.

With decreasing the surface of the mold cavity, there decreases the contact area of the poured metal with this surface, through which the heat from the metal is removed into the sand environment. This improves the fillability of the mold by the metal.

For the same reasons the minimal surfaces principle is effective for designing patterns of gating systems. Oval 
outlines of the casting reduce the probability of cracking because of the hindered shrinkage of the casting.

On the website [4] it is indicated that the gyroid model is built using the freely available software Surface Evolver, created by Kenneth Brakke. The search of the minimum area surface or the total energy of the surface tension is only the simplest application of this software.

With its help, when designing castings or optimizing the constructions to improve their processability for any type of molding, it is possible to build the "minimal" surfaces in terms of more complex functionals. Such functional can be a combination of surface tension energy, gravitational energy, flexural deformation energy, etc., as well as arbitrary userdefined surface integrals [7]. This makes it possible to obtain not only the drawing of the pattern with the MS on the computer, but also the MS in combination with the specified service characteristics of the casting.

In particular, during LFC such drawing can be sent directly to a computer-controlled 3D-mill. The latter in the automatic mode will produce the casting pattern or the pattern of its press mold from the foam blocks, for example, from two halves, for the further serial production of casting patterns or their parts with the help of an autoclave or an automatic formation machine.

Surface Evolver, when designing castings, can process objects with arbitrary topology, which are subordinate to various volumetric and boundary connections.

For example, it is possible to fix the volume covered by the surface (isoperimetric problems) or the contact angle on the lines of intersection of the desired surface with some given surface.

For searching the configuration with the minimum energy, this software uses the gradient descent method. It is one of the examples of software that can be used for the MS construction and their combination with other surfaces when engineering the casting patterns or gating systems for various casting processes [7].

Minimal surfaces are analytical, i.e. each of their points in space is determined by an analytical function. This improves processability of: designing and producing the patterns, as well as the equipment for them; the pattern quality control; the casting quality control; the further casting processing with the help of modern equipment with software.

Investigation of the possibility of copying the crystal lattice structure in cast frame cellular constructions using volumetric prefabricated structures with repetitive unified elements [1] has the following sequel. In modern crystallography, when summarizing new studies, the increasing attention is paid not only to traditional crystal structures but also to quasicrystals. Professor Dan Shechtman was awarded the Nobel Prize in Chemistry 2011 for the discovery of quasicrystals.

The structural perfection of thermodynamically stable quasicrystals puts them on a par with the best of ordinary crystals and allows to classifying them as a subclass of crystals. Also, artificial materials with a structure similar to the structure of quasicrystals were created and they were called quasicrystalline metamaterials. Quasicrystals have very low thermal conductivity and a low coefficient of friction.

A model of the quasicrystal structure may be created based on the Penrose tiling with two "elementary cells" connected to each other according to definite rules of matching [8]. At present, the three-dimensional generalization of the Penrose tiling is being developed, which is the Ammann-Mackay structure. It is composed of narrow and wide rhombohedrons, i.e. hexahedral figures, each face of which is a rhombus.

The "thin" and "thick" rhombuses from the Penrose tiling are shown in Fig. 2, above. In Fig. 2, below, one can see the drawings of the frame patterns of rhombohedrons, filling the space by which analogously to the quasicrystal structure gives the spatial pattern [9]. The examples of ways of mounting for similar frame cellular patterns are shown in [1].

Investigations of quasicrystals stimulated the revival of interest in the ideas and methods of constructing mosaics, which were the subject of study of the mathematical theory, called the "theory of tilings of unbounded plane or volume" [8]. This theory studies isoperimetric problems for polyhedra, finite and infinite partitions into rhombuses, periodical minimal surfaces, 3D-puzzles, fractal trees and others.

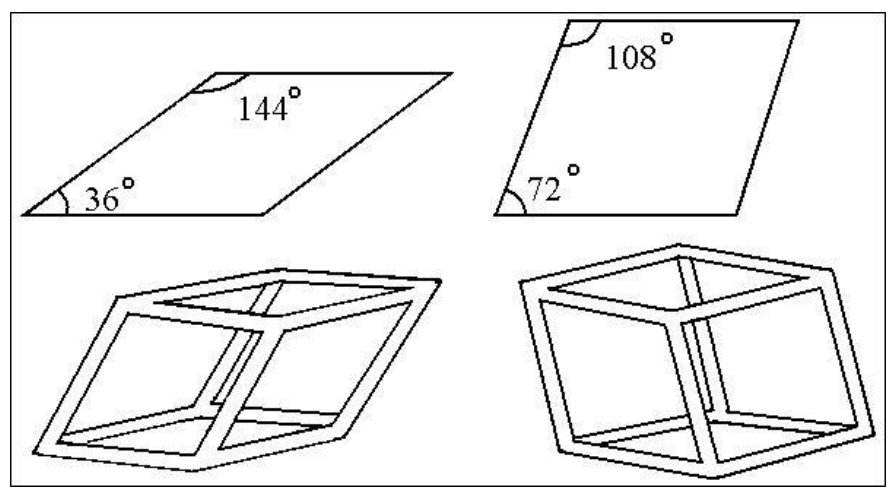

Fig. 2. Above: Rhombs from the Penrose tiling; Below: The frame patterns of rhombohedrons.

In particular, the computer graphics mastered the problems of tiling by the method of curves or surfaces of Bezier, who used them for computer-aided design of car bodies.

Technologies of software and virtual construction of spatial structures are intensively developing. Description of quasiperiodical structures is formed based on the integration of different disciplines, such as modern geometry, number theory, statistical physics, and also the concept of the golden ratio.

The unexpected appearance of the golden ratio in the structure of quasicrystals indicates the presence of the "living motive" in their symmetry, as far as, in contrast to non-living crystals, only living world accepts outstanding proportions of the golden ratio [8].

Fig. 3 shows the examples, taken from the open Internet sources, with the computer optimization of the construction of six castings. 

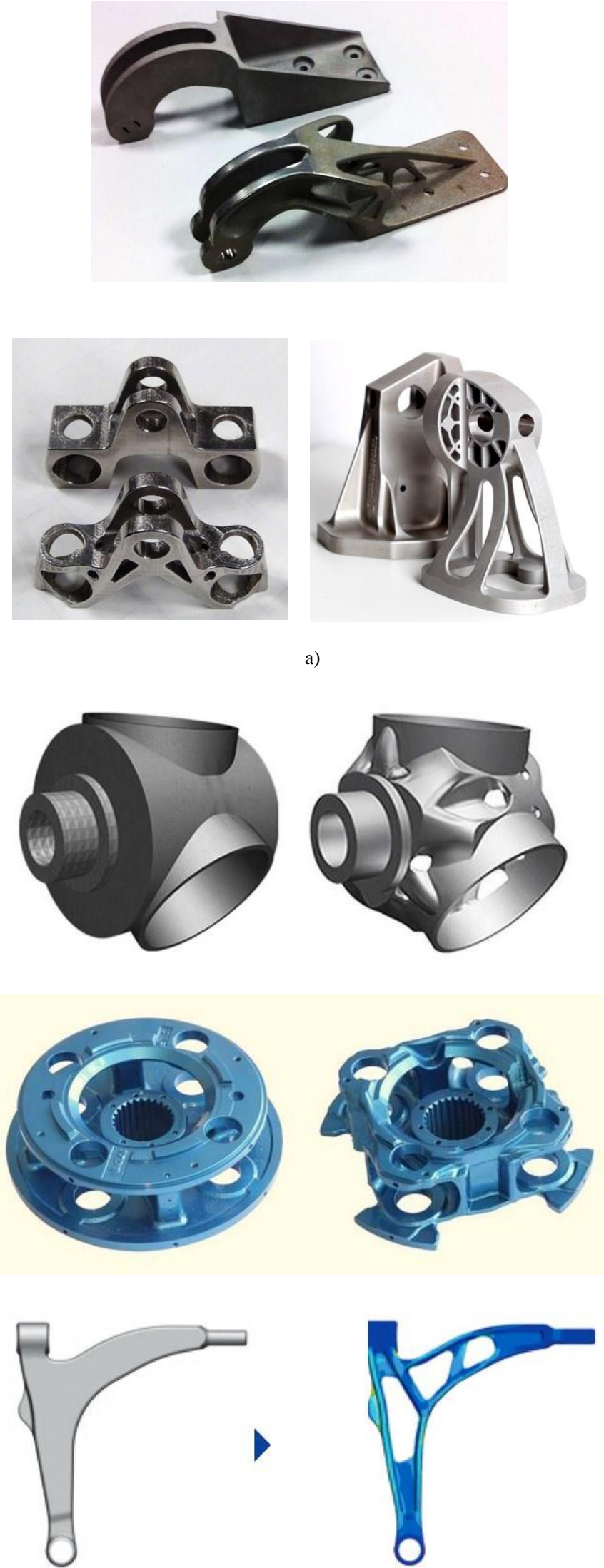

b)

Fig. 3. Examples of computer optimization of the casting constructions.

In Fig. 3a) serial castings are presented at the top of the photo, and the designs of castings, "proposed" by computer, are shown below. Most of such castings are meant for mounting in the movable structures.

In Fig. 3b) in each couple of images the serial casting is presented on the left, and the casting design, which is computer-optimized according to the requirements for its use, is shown on the right.

Thus, one can see how traditional monolithic constructions "turn" into the elegant frame cellular ones, serving as the illustrative examples of metal saving and appearance improving. The new constructions can be easily carried out by the LFC method.

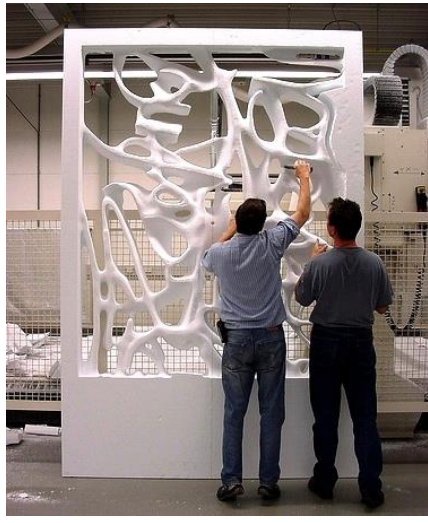

a)

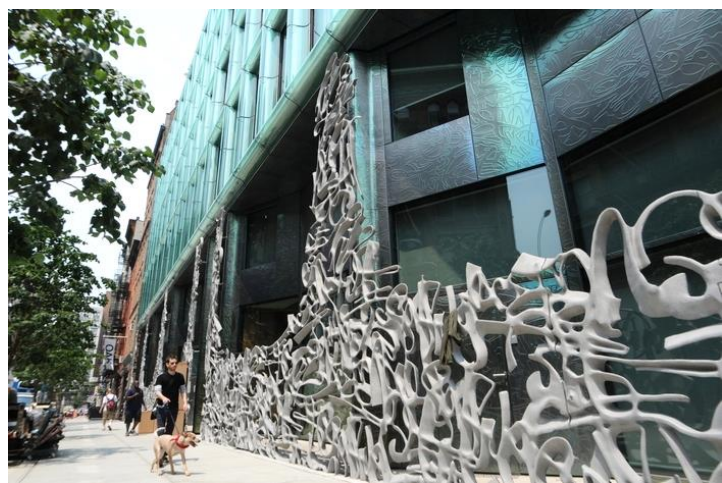

b)

Fig. 4. Examples of art castings as the architectural decoration: a) the gate pattern; b) sections of aluminium castings of the collage, assembled along the large building in New York.

The above mentioned 3D-mills are increasingly used for the production of single casting patterns from foam.

The examples of large-size patterns for art castings are presented in Fig. 4. Using such patterns, the "graffiti" collage was cast from aluminium as the architectural decoration of the many meters size for the apartment house in the luxury block of New York (the project Herzog \& de Meuron, 2006). The prefabricated collage along the front side of the house consists of dozens of castings, including several gates, designed with the help of computer technologies with the thickness of walls, which is optimum for the casting process. In Fig. 4a) it is shown that the pattern of gates and the details of the 3D-mill 
can be seen in the background. In Fig. 4b) sections of aluminium castings of the collage are presented.

A new step in obtaining metal products is their production by 3D-printers. The cellular constructions, obtained by 3D-printers according to computer programs, can have quite a fantastic look as it is shown, for example, in Fig. 5, taken from the open Internet sources.
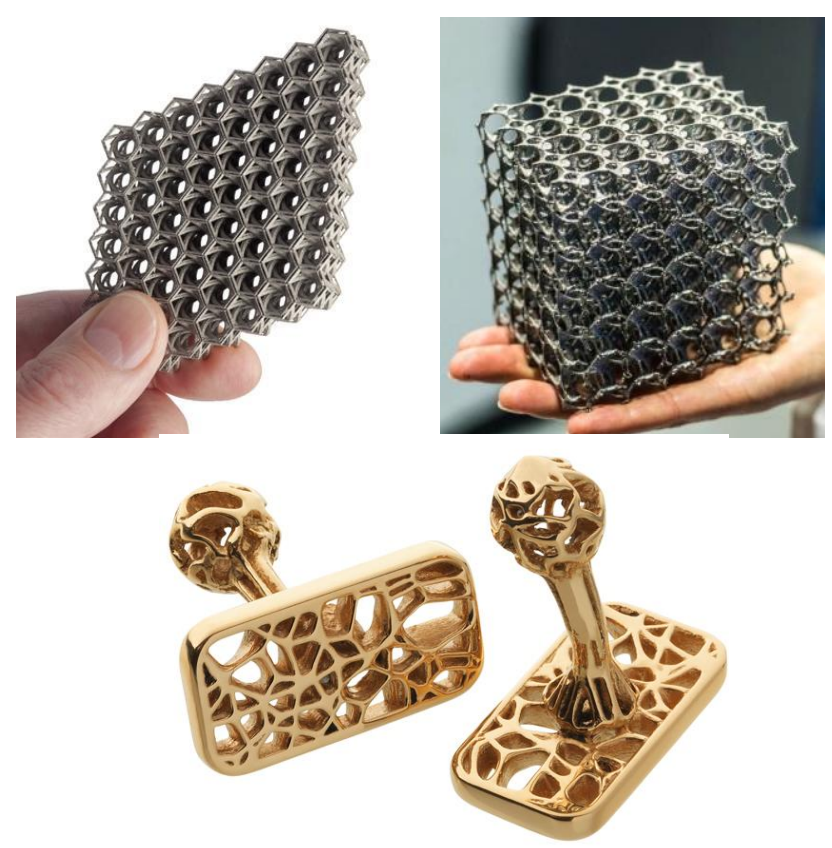

Fig. 5. Examples of constructions obtained by 3D-printing according to the computer programs.

Cellular castings can inherit properties of the nature structures and also can be created by human imagination with the help of computer design according to the human requirements. This significantly expands the existing range of metal products.

\section{REFERENCES}

[1] V. S. Doroshenko, "Methods of Obtaining Frame and Cellular Cast Materials and Details by Gasified Patterns," (in Russian), Liteinoye Proizvodstvo, vol. 9, pp. 28-32, 2008.

[2] A. R. Oganov, A. O. Lyakhov, and M. Valle, "How Evolutionary Crystal Structure Prediction Works - and Why," Acc. Chem. Res., vol. 44, pp. 227-237, 2011. http://dx.doi.org/10.1021/ar1001318

[3] A. H. Schoen, Infinite Periodic Minimal Surfaces without SelfIntersections. NASA Technical Note TN D-5541, Washington, D. C., 1970.

[4] Bathsheba Sculpture [Online] Available: http://www.bathsheba.com/math/gyroid/ [Accessed: Aug. 5, 2014].

[5] University of Ulm, Institute of Polymer Science, Germany [Online] Available: http://polymer.physik.uni-ulm.de/mol_graphics/ [Accessed: Aug. 5, 2014].

[6] V. S. Doroshenko, "Spatial Cast Constructions, Obtained in a Volume of Sand (in Russian)," Metal and Casting of Ukraine, vol. 7-8, pp. 45-52, 2009.

[7] V. Meshkov, "Minimal Surfaces and Surface Evolver of Kenneth Brakke (in Russian)," Vestnik Molodykh Uchenykh, vol. 1, p. 84, 2004.

[8] V. S. Belyanyn, "Quasicrystals and the Golden Ratio," (in Russian) Nauka i Zhizn, vol. 10, pp. 68-76, 2005.

[9] V. S. Cherednychenko, Materials Science. Technology of Construction Materials (in Russian). 2nd ed., Moscow: Omega-L, 2006.

[10] V. S. Doroshenko, "Mathematical Design of Frame-Cellular Castings (in Russian)," Liteinoye Proizvodstvo, vol. 2, pp. 9-12, 2013.

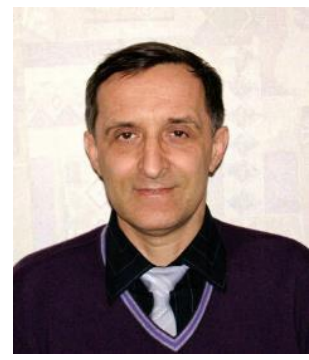

Doroshenko Volodymyr received the Doctor's degree in Technical Sciences in 1990 from Kyiv Polytechnical Institute, Ukraine. The title of the Thesis was "Obtaining of Castings of Hydrodistributors from Cast Iron with Globular Graphite in Vacuum-processed Molds". Since 1982 he has been an Engineer (speciality: Foundry of Ferrous and Nonferrous Metals). He is currently a Senior Research Fellow with Physico-Technological Institute of Metals and Alloys, Ukraine.

Major areas of his scientific activity are: heat and mass transfer, gas and hydrodynamics of the interaction of a melt and a crystallizing metal with a sand casting mold, physics and chemistry of processes of sand casting molds production, particularly with the use of vacuumization; new methods of forming for foundry, which ensure environmental safety of production and improve the quality of metal castings; methods of casting into vacuum-processed molds; use of cryo-technologies in foundry, in particular, technological bases of accurate blanks obtained by single-use ice models. $\mathrm{He}$ is the author of more than 335 scientific and technical papers, including t 80 patents for inventions in Ukraine and Russia.

E-mail: dorosh@inbox.ru

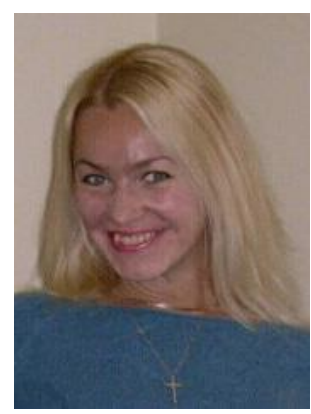

Mul Olena received the Doctor's degree in Physics and Mathematics in 2001 from the Space Research Institute of NASU-NSAU, Kyiv, Ukraine. The title of her Thesis was "Analysis of Self-Oscillation Processes in the Complex Continuous and Discrete Systems". Since 1994 she has been an Engineer-mathematician (speciality: Applied Mathematics). She is currently an Associate Professor with the Computer-Integrated Department of Ternopil National Ivan Puluj Technical University.

Major areas of her scientific activity are: control theory on time scales with special emphasis on hybrid systems; controllability and optimality of nonlinear control systems; analysis and control of dynamical systems; numerical analysis; perturbation theory; theory of vibrations; mathematical physics and computational mathematics for nonlinear boundary value problems in PDE's and ODE's.

She is a member of IAMP.

She has received grants from INTAS, IUPAP, NATO, ESF, UNESCO, CIME, C.I.R.M., the Banach Center (Warsaw, Poland), Max Planck Institute of Physics of Complex Systems (Dresden, Germany) as well as 3-year FCT Post-doctoral fellowship "Analysis of Vibrations in Nonlinear Dynamical Systems" at Department of Mathematics, University of Aveiro, Portugal (2004-2007); 6 months scientific fellowship "Numerical and Asymptotical Methods for Highly Oscillatory Differential Equations" at the Department of Applied Mathematics and Theoretical Physics, Centre for Mathematical Sciences, University of Cambridge, UK (2012).

E-mail: ms.olena@gmail.com

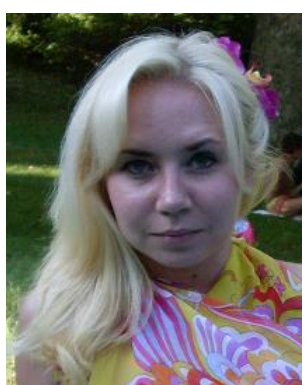

Kravchenko Olena is a post-graduate student with International Research and Training Center for Information Technologies and Systems of NASU-MESU, Kyiv, Ukraine. She is a mathematician-programmer (speciality: Applied Mathematics). She is currently a software engineer with Glushkov Institute of Cybernetics of NASU, Ukraine.

Major areas of her scientific activity are: group method of data handling (GMDH) and other methods of inductive modeling under assumptions; robotics problems in using a mobile robot manipulator for repair and rescue works under extreme conditions of catastrophic situations in underground mining constructions; ecological problems in development of geoinformation systems for the prediction of natural meteorological disasters; interval method of telemetry information reliability control for monitoring the casting cooling in a sand mold; mathematical methods for assessing the quality of cast iron by temporal experimental data; monitoring and geoprocessing of the earth surface probing processes. She is the author of about 20 papers, published in scientific journals and presented at international and Ukrainian scientific conferences.

E-mail: len327@ukr.net 